\title{
THE CLASSIFICATION OF FREE ACTIONS OF CYCLIC GROUPS OF ODD ORDER ON HOMOTOPY SPHERES
}

\author{
BY WILLIAM BROWDER, ${ }^{1}$ TED PETRIE AND C. T. C. WALL
}

\author{
Communicated by M. F. Atiyah, June 19, 1970
}

Consider the cyclic group $Z_{p}$ acting freely on a homotopy sphere $\Sigma^{2 n+1}$. This action is a map $\mu: Z_{p} \times \Sigma \rightarrow \Sigma$. We shall consider the three cases where the action is smooth, piecewise linear (PL) or topological. If we pick a generator $T \in Z_{p}$ then we may say that two actions $\mu_{i}: Z_{p} \times \Sigma_{i}^{2 n+1} \rightarrow \Sigma_{i}^{2 n+1}, i=1,2$, are equivalent actions if there is an equivalence $f: \Sigma_{1} \rightarrow \Sigma_{2}$ in the appropriate category (i.e. $f$ is diffeomorphism, PL equivalence or homeomorphism depending on whether $\mu_{i}$ are smooth, PL or topological) such that $f \mu_{1}(T, x)=\mu_{2}(T, f(x))$. Among smooth actions we have the linear actions on $S^{2 n+1} \subset C^{n+1}$ where the action is given by

$$
\left(z_{0}, \cdots, z_{n}\right) \rightarrow\left(\exp \left[2 \pi i \theta_{0} / p\right] z_{0}, \cdots, \exp \left[2 \pi i \theta_{n} / p\right] z_{n}\right)
$$

where $\theta_{0}, \cdots, \theta_{n}$ are integers between 1 and $p-1$, prime to $p$. The quotient of $S^{2 n+1}$ by this action is the Lens space $L^{2 n+1}\left(p ; \theta_{0}, \cdots, \theta_{n}\right)$, and it is well known that for any free $Z_{p}$ action on $\Sigma^{2 n+1}$, the orbit space $\Sigma / Z_{p}$ is homotopy equivalent to $L^{2 n+1}\left(p ; \theta_{0}, \cdots, \theta_{n}\right)$ for some appropriate choice of $\theta_{0}, \cdots, \theta_{n}$.

Now two $Z_{p}$-actions $\left(\mu_{i}, \Sigma_{i}\right), i=1,2$, are equivalent if and only if the orbit spaces $\Sigma_{i} / Z_{p}$ are equivalent in the appropriate category. The equivalence is given by an orientation preserving isomorphism $g: \Sigma_{1} / Z_{p} \rightarrow \Sigma_{2} / Z_{p}$ such that $g_{*}(T)=T$ where $T$ is identified with the corresponding element in $\pi_{1}\left(\Sigma_{i} / Z_{p}\right)$. Thus the problem of equivalence of $Z_{p}$ actions is the problem of classifying manifolds of the homotopy type of Lens spaces up to equivalence in the corresponding category.

In this note we announce a procedure for doing this when $p$ is odd, $n \geqq 2$. Some related work has been done by $\mathrm{R}$. Lee [3], [4]. The result for $p=2$ was previously found by Lopez de Medrano [5] and Wall [11] and the answer for $p$ odd is quite analogous. One uses the theory of surgery to study the problem, and calculates as far as possible the terms arising. As usual one arrives at a complete classification in the

AMS 1970 subject classifications. Primary 57D65, 57E05; Secondary 20C10.

Key words and phrases. Free cyclic group actions on spheres, surgery, free action, Lens space, simple homotopy structure, signature invariant, torsion, suspension.

${ }^{1}$ Partially supported by an NSF grant. 
$\mathrm{PL}$ and topological categories but the smooth case must await more profound knowledge of the homotopy of spheres.

We recall [9] that a (simple) homotopy structure on a manifold $X^{n}$ is a pair $\left(M^{n}, f\right), M^{n}$ a manifold in the appropriate category, $f: M^{n} \rightarrow X^{n}$ a (simple) homotopy equivalence. Two structures $\left(M_{i}, f_{i}\right), i=0,1$, on $X$ are concordant if there is a structure $\left(W^{n+1}, F\right)$ on $X \times[0,1], F: W \rightarrow X \times[0,1]$ such that $\partial W$ is isomorphic to $M_{0} \cup M_{1}$ (in the category) and $F \mid M_{i}=f_{i}$ into $X \times i, i=0,1$. The set of concordance classes of structures is denoted by $s_{\mathrm{e}}^{\epsilon}(X)$ where $\epsilon=h$ or $s$ (for homotopy or simple homotopy structures), $\mathfrak{e}=$ Diff, PL or Top is the category of manifolds. It follows from the $s$-cobordism theorem that $\left(M_{0}, f_{0}\right)=\left(M_{1}, f_{1}\right)$ in $\delta^{s}(X)$ if and only if $f_{1}^{-1} f_{0}$ is homotopic to an isomorphism, provided the dimension of $X=n \geqq 5$.

The endproduct of the theory of "Surgery on maps" is the following exact sequence:

(S) $\rightarrow L_{n+1}^{\bullet}(\pi) \stackrel{\omega}{\rightarrow} S^{\bullet}(X) \stackrel{\eta}{\rightarrow}[X, G / H] \stackrel{\sigma}{\rightarrow} L_{n}(\pi) \quad$ where $\epsilon=h$ or $s$,

$H=0, \mathrm{PL}$ or Top depending on whether we are in the Diff, PL or Top category, $S$ is structures in that category, $\pi=\pi_{1}(X)$, and $L_{k}^{e}(\pi)$ is a covariant functor on finitely presented groups into abelian groups and $L_{n+1}^{e}(\pi)$ acts on $\oint^{\epsilon}(X)$ to make the sequence exact in the strong sense.

There is a natural relationship between this exact sequence and the representation theory of $\pi$. Starting from the complex representation ring $R(\pi)$ of $\pi$ we form the "localized ring" $S^{-1} R(\pi)$, where $S \subset R(\pi)$ is the multiplicative set $\left\{\lambda^{k} \mid \lambda=N-R, k=0,1,2 \cdots\right\}, N$ is the order of $\pi$ and $R$ is the regular representation of $\pi$. There is a commutative diagram [8]

$$
\begin{aligned}
& \cdots \rightarrow L_{n+1}^{\bullet}(\pi) \stackrel{\omega}{\rightarrow} s^{e}(X) \stackrel{\eta}{\rightarrow}[X, G / H] \stackrel{\sigma}{\rightarrow} L_{n}^{e}(\pi) \\
& \begin{array}{ll}
\downarrow x & \downarrow A \\
R(\pi) \rightarrow S^{-1} R(\pi) \rightarrow S^{-1} R(\pi) / R(\pi)
\end{array}
\end{aligned}
$$

when $\pi$ is finite and $n$ is odd.

We now restrict attention to the case $\pi=Z_{p}$ and $X$ is the orbit space of a free $Z_{p}$ action on a homotopy sphere. Then $X$ is homotopy equivalent to a Lens space (but not necessarily simple homotopy equivalent to one). We now discuss the sequence (T) with $X$ $=L^{2 n+1}\left(p ; \theta_{0}, \cdots, \theta_{n}\right)$, and $\pi=\pi_{1}(X)=Z_{p}$. It follows that elements of $\mathcal{S}^{s}\left(L^{2 n+1}\left(p ; \theta_{0}, \cdots, \theta_{n}\right)\right)$ are in $1-1$ correspondence with equivalence classes of $Z_{p}$ actions whose orbit space is simple homotopy equivalent 
to $L^{2 n+1}\left(p ; \theta_{0}, \cdots, \theta_{n}\right)$ and all other actions may be obtained from these by action of the group $L_{2 n}^{h}\left(Z_{p}\right)$ and the Whitehead group $\mathrm{Wh}\left(Z_{p}\right)$ (on the orbit space).

Theorem 1 [2]. For $X=L^{2 n+1}\left(p ; \theta_{0}, \cdots, \theta_{n}\right), p$ odd $n \geqq 2, \epsilon=s$ or $h$ the map $\eta$ is onto.

THEOREM 2 ([7] AND [12]). $L_{2 k}^{s}\left(Z_{p}\right)_{0}$ is a free abelian group of rank $\frac{1}{2}(p-1)$ and acts freely on $s^{s}(X)$ for any $X$ with $\pi_{1}(X)=Z_{p}$ (in each category) $k \geqq 3$; moreover, the image of $\chi$ in $R(\pi)$ is of rank $\frac{1}{2}(p-1)$.

Here $L_{n}^{s}(\pi)_{0}=\operatorname{kernel}\left(L_{n}^{s}(\pi) \rightarrow L_{n}^{s}(0)\right)$.

The map $A$ can be described in several different but related ways:

(a) As a signature invariant generalizing the $Z_{2}$-signature used in [11].

(b) As a signature invariant of the type defined by Atiyah-Singer as in [7].

(c) As an element of $K O\left(B Z_{p}\right)$ using the $K O$ theory orientation of PL bundles defined and exploited by Sullivan [10].

Let $[M, f] \in \mathscr{S}^{\epsilon}\left(L^{2 n+1}\left(p ; \theta_{0}, \cdots, \theta_{n}\right)\right)$ and if $\Sigma$ is the total space of the $Z_{p}$ bundle over $M$ induced by $f$, then some multiple $p^{k} \Sigma$ bounds a manifold $W$ supporting a free action of $Z_{p}$. Set $A[M, f]=(p-R)^{-k}$ $\cdot \operatorname{Sgn}\left(Z_{p}, W\right)$ where $\operatorname{Sgn}\left(Z_{p}, W\right)$ is the representation of $Z_{p}$ constructed from the $Z_{p}$ invariant bilinear form on $\hat{H}^{n+1}(W, \boldsymbol{C})$ defined by the cup product pairing (see [1]).

In particular one may show that the definition of $A$ does not depend on the choice of homotopy equivalence. Namely, if $\left(M_{i}, f_{i}\right)$ represent elements $x_{i} \in \delta\left(L^{2 n+1}\left(p ; \theta_{0}, \cdots, \theta_{n}\right)\right), i=1,2$, and if there is an orientation preserving PL (topological) equivalence $g: M_{1} \rightarrow M_{2}$ such that $g_{*}(T)=T\left(\right.$ in $\left.\pi_{1}\left(M_{i}\right)=Z_{p}\right)$ then $A\left(x_{1}\right)=A\left(x_{2}\right)$.

Theorems 1 and 2 together give one a classification theorem of sorts in each category. For the categories PL and Top, G/PL and G/Top are amenable to calculations. Namely from the work of Sullivan [10] in the PL case, which for odd primes the work of Kirby-Siebenmann shows is equivalent to the Top case, we have:

Theorem 3. If $X=L^{2 n+1}\left(p ; \theta_{0}, \cdots, \theta_{n}\right), p$ odd, then $[X, G / H]$ $\cong \tilde{K} O(X)$, where $H=\mathrm{PL}$ or Top.

ThEOREM 4. The invariant $A$ characterizes the PL or topological type of a simple homotopy structure on $L^{2 n+1}\left(p ; \theta_{0}, \cdots, \theta_{n}\right), n \geqq 2$, i.e. if $\left(M_{i}, f_{i}\right) \in S_{\mathrm{PL}}^{s}\left(L^{2 n+1}\left(p ; \theta_{0}, \cdots, \theta_{n}\right)\right), n \geqq 2, i=0,1$, then $\left(M_{0}, f_{0}\right)$ $=\left(M_{1}, f_{1}\right)$ if and only if $A\left(M_{0}, f_{0}\right)=A\left(M_{1}, f_{1}\right)$.

Following Milnor [6, pp. 404-406] one can define a torsion in- 
variant $\Delta$ for a manifold $M=\Sigma^{2 n+1} / Z_{p}$, where $\Delta(M) \in Q\left(Z_{p}\right) / \Sigma$, the rational group ring modulo the ideal generated by the sum of the group elements. If $\Delta(M)=\Delta\left(M^{\prime}\right)$ then $M$ and $M^{\prime}$ are simple homotopy equivalent. Furthermore:

TheORem 5 [12]. Two actions of $Z_{p}$, $p$ odd, on homotopy $(2 n+1)$ spheres, $n \geqq 2$, are PL or topologically equivalent if and only if their invariants $A$ and $\Delta$ agree.

One may also describe which pairs $\Delta$ and $A$ arise as the invariants of such $Z_{p}$ actions but we will not do this here.

Let $\mu: Z_{p} \times S^{2 n+1} \rightarrow S^{2 n+1}$ be a free PL or topological action on the sphere. We may define the suspension $\Sigma \mu$, of $\mu$ by taking the induced action on the join $S^{2 n+1} * S^{1}$, using the action $T\left(e^{2 i \pi t}\right)=e^{2 i \pi(t+(1 / p))}$ on $S^{1}$. Then $\Sigma \mu$ is a free PL or topological action on $S^{2 n+3}$ and if the orbit space of $\mu=S^{2 n+1} / Z_{p}$ is homotopy equivalent to $L^{2 n+1}\left(p ; \theta_{0}, \cdots, \theta_{n}\right)$ then the orbit space of $\mu_{\theta}, S^{2 n+3} / Z_{p}$ is homotopy equivalent to $L^{2 n+3}\left(p ; \theta_{0}, \cdots, \theta_{n}, 1\right)$.

Using Theorem 5 and studying the effect of suspension on $A$ and $\Delta$ one obtains:

COROLLARY 1 [12]. If $n>2$, then every PL or topological action on $S^{2 n+1}$ is the suspension of a unique action on $S^{2 n-1}$.

Note that the suspension of a smooth action $\mu: Z_{p} \times \Sigma^{2 n+1} \rightarrow \Sigma^{2 n+1}$ will be smoothable if and only if the orbit space of the diagonal action of $Z_{p}$ on $\Sigma^{2 n+1} \times S^{1}$ is diffeomorphic to $S^{2 n+1} \times S^{1}$, which is equivalent to the condition that $\Sigma^{2 n+1}$ is diffeomorphic to $S^{2 n+1}$. In that case there may be many smoothings of it.

Corollary 2. Let $\mu: Z_{p} \times \Sigma^{2 n+1} \rightarrow \Sigma^{2 n+1}$ be a smooth free action, $n>2$. Then $\mu$ is a smooth suspension of a smooth action on $S^{2 n-1}$.

This follows from Corollary 1 using the smoothing theory of PL manifolds.

If we consider the problem of comparing actions in the different categories, we first note as above that using the theorem of KirbySiebenmann, since $p$ is odd there is a 1-1 correspondence between PL and topological actions on spheres of dimension 5 or more. The question of smoothing PL actions can be completely solved using results of Sullivan on the homotopy of $G / \mathrm{PL}$ and its relation to $G / O$ in the "world of odd primes." In particular for $p=3$ or 5 every free PL $Z_{p}$ action can be smoothed. However for $p=7$ there is a free PL action of $Z_{p}$ on $S^{9}$ which cannot be smoothed, namely the suspension of a $Z_{p}$ action on the generator $\Sigma^{7}$ of $\Gamma^{7}$, which may be constructed using 
the equation for the Brieskorn sphere $z_{0}^{5}+z_{1}^{3}+z_{2}^{2}+z_{3}^{2}+z_{4}^{2}=0,\|z\|=1$, and the action

$$
T\left(z_{0}, \cdots, z_{4}\right)=\left(\lambda^{6} z_{0}, \lambda^{10} z_{1}, \lambda^{15}\left(z_{2}, z_{3}, z_{4}\right)\right), \quad \text { where } \lambda^{7}=1 .
$$

\section{REFERENCES}

1. M. F. Atiyah and I. M. Singer, The index of elliptic operators. III, Ann. of Math. (2) 87 (1968), 546-604. MR 38 \#5245.

2. W. Browder, Free $Z_{p}$-actions on homotopy spheres, Proc. Georgia Topology Conference (to appear).

3. R. Lee, Piecewise linear classification of some free $Z_{p}$-actions on $S^{4 k+8}$, Michigan Math. J. 17 (1970), 149-160.

4. - On the Wall group $L_{4 k+3}\left(Z_{p}\right)$ (to appear).

5. S. Lopez de Medrano, Some results on involutions of homotopy spheres, Proc. Conference Transformation Groups (New Orleans, La., 1967) Springer-Verlag, New York, 1968, pp. 167-174; Thesis, Princeton University, Princeton, N. J., 1968.

6. J. Milnor, Whitehead torsion, Bull. Amer. Math. Soc. 72 (1966), 358-426. MR 33 \#4922.

7. T. Petrie, The Atiyah-Singer invariant, the Wall groups $L_{n}(\pi, 1)$ and the function $t e^{x}+1 / t e^{x}-1$, Ann. of Math. (2) 92 (1970), 174-187.

8. - Representation theory, surgery, and free actions of finite groups on varieties and homotopy spheres, Proc. Conference in Honor of N. Steenrod, Springer-Verlag, New York (to appear).

9. D. Sullivan, Triangulating homotopy equivalences, Thesis, Princeton University, Princeton, N. J., 1966.

10. - Geometric topology, M.I.T. Press, Cambridge, Mass., 1970 (lecture notes).

11. C. T. C. Wall, Free piecewise-linear involutions on spheres, Bull. Amer. Math. Soc. 74 (1968), 554-558.

12. - Surgery of compact manifolds, Academic Press, New York (to appear).

Princeton University, Princeton, New Jersey 08540

Institute for Defense Analyses, Princeton, New Jersey 08540

UNIVERSITY OF LIVERPOOL, LIVERPOOL, L693BX ENGLAND 